\title{
A NOTE ON PARAMETRIC AVAILABILITY
}

\author{
M.S. FINKELSTEIN \\ Department of Mathematical Statistics \\ University of the Orange Free State \\ PO Box 339, 9300 Bloemfontein, Republic of South Africa \\ (e-mail:msf@WWG3.uovs.ac.za)
}

\begin{abstract}
Availability of a binary repairable system is generalised on the case when the quality of the system's operation is characterised by some decreasing in time (since the latest renewal) function of performance. The corresponding equation for the time-dependent availability is derived and the stationary characteristic is considered as well. The probability of exceeding the fixed level of performance for an arbitrary instant of time is also obtained. A further generalisation is performed by assuming the possibility of a gradual repair. This means that the system can be partly available (with the decreased level of performance) during the process of repair. Finally, the specific case of imperfect repair is treated. Possible generalisations of the last approach are discussed.
\end{abstract}

Keywords: Availability, Function of performance, Gradual repair, Imperfect repair.

\section{INTRODUCTION}

Consider a repairable system with $F(x)(G(x))$ as the distribution function (DF) of time to failure (repair). Let the system starts functioning at $t=0$ and then the usual alternating renewal process pattern takes place: after the first repair (renewal) the system is "as good as new" $(F(x))$ and the time of repair after the second failure is described by the same $G(x)$ etc. Availability $A(t)$ is usually defined [1] as probability that the system is functioning at an arbitrary instant of time $t$. A following equation for the stationary availability $A_{S}$ as $t \rightarrow \infty$ is well known:

$$
A_{S}=\frac{T}{T+\tau},
$$

where $T$ is the mean time to failure and $\tau$ is the mean time of repair. 
Thus, availability describes the binary case when the system can be only in the state of operation or repair. In other words, if $X_{t}$ denotes the corresponding binary variable, then $A(t) \equiv E\left[X_{t}\right]$. Generalisation can be performed in various directions. In this paper a simple parametric model will be treated.

Let the operating system be characterised by some function of performance $\Phi(x)$, where $x$ is the time elapsed since the last renewal. It is often a decreasing function of time and this is quite natural for describing degradation in system's performance. In applications $\Phi(x)$ can characterise, for instance, the decreasing in time accuracy of the information measuring system or effectiveness of some process of production. Usually this is due to some type of wearing out, which often takes place in the process of functioning. Assume that $\Phi(x)$ is a deterministic function, though the approach can be easily generalised for stochastic processes. Thus, immediately after each repair, the function of performance is $\Phi(0)$ and it is assumed to be equal to 0 while the system is repaired.

Denote by $\Phi_{t}$ the random value of this function at time instant $t$ from the start. This means that $\Phi_{t}=0$, if the system is being repaired at this moment and $\Phi_{t}=\Phi(x)$, if it is operating (and $x$ is the time elapsed since the last renewal). It is absolutely straightforward from the results of the theory of (alternating) renewal processes [1], that

$$
E\left[\Phi_{t}\right]=(1-F(t)) \Phi(t)+\int_{0}^{t} h(x)[1-F(t-x)] \Phi(t-x) d x
$$

where $h(x)$ is the renewal density function of the renewal process without delay, governed by the DF which is the convolution of $F(x)$ and $G(x)$. If $\Phi(x) \equiv 1$, then we arrive at the case of availability. Hence, equation (1) generalises the notion of conventional availability to be called "parametric availability" and will be denoted as $A_{P}(t) \equiv E\left[\Phi_{t}\right]$. Similar to $A(t)$, which is the mean of binary variable, $A_{P}(t)$ is the mean of the continuous one. It defines the expected quality of system's performance at an arbitrary instant of time $t$ and can be considered as its important characteristic.

Applying the key renewal theorem [1] to equation (1) as $t \rightarrow \infty$, the stationary parametric availability can be obtained in a usual way: 


$$
A_{S P}=\frac{1}{T+\tau} \int_{0}^{\infty}(1-F(x)) \Phi(x) d x
$$

where $T$ and $\tau$ are the mean times to failure and repair, respectively.

It should be noted, that unlike the binary case, parametrical availability is well defined for the instantaneous repair when $\tau=0$.

The probability of $\Phi_{t}$ exceeding the fixed level $\Phi_{0}: \Phi(\infty)<\Phi_{0}<\Phi(0)$, can be easily derived for the monotonically decreasing $\Phi(x)$ :

$$
P\left(\Phi_{t} \geq \Phi_{0}\right)=(1-F(t)) \mathrm{I}\left(t_{0}-t\right)+\int_{0}^{t} h(x)[1-F(t-x)] \mathrm{I}\left(x-t+t_{0}\right) d x,
$$

where $\mathrm{I}(x)$ is an indicator function: $\mathrm{I}(x)=1$ for $x \geq 0$; $\mathrm{I}(x)=0$ for $x<0$ and $t_{0}$ is uniquely defined from the equation: $\Phi\left(t_{0}\right)=\Phi_{0}$.

Similar to (2), as $t \rightarrow \infty$ we can derive the equation for the stationary value:

$$
P_{S P}=\frac{1}{T+\tau} \int_{0}^{t_{0}}(1-F(x)) d x .
$$

\section{Example 1}

Let $\quad F(x)=1-\exp \{-\lambda x\}, \Phi(x)=1-\exp \{-\alpha x\}, \tau=0$. Then

$$
A_{S}=\frac{\lambda}{\lambda+\alpha}, \quad P_{S P}=\lambda \int_{0}^{-\frac{\ln \Phi_{0}}{\alpha}} \exp \{-\lambda x\} d x=1-\Phi_{0}^{\frac{\lambda}{\alpha}}
$$

In the following sections of this paper we shall discuss two possible generalisations of the parametric availability approach.

\section{GRADUAL REPAIR}

While considering operation of repairable (parametric) systems in practice, one can notice that some types of systems can be partly operable during the process of repair. It takes place, for instance, when after rather quick replacement of the failed part or of the whole system, the process of adjustment (alignment) is performed. During this process the quality of the potential performance is monotonically increasing in order to reach finally the "ideal" level 
$\Phi(0)$. If the demand for using the system at this stage occurs, then some types of systems are capable to start operating with reduced quality. And this is better than nothing.

A practical example of this situation is the high accuracy onboard inertial navigation system, when after the failure and replacement of the gyroscope at least several hours (and sometimes more) are needed to reach the nominal accuracy of obtaining navigational parameters. And the procedure is gradual, which means that the accuracy is monotonically increasing in the process of this operation We had applied the results of this paper on gradual and imperfect repair to reliability and availability case study of the Russian precise inertial submarine navigation system. The accuracy in this example is measured by the navigational error $\sigma(x)$, which is the increasing function of $x$. This means that by increasing accuracy we decrease the error: $\Phi(x)=(\sigma(x))^{-1}, \Phi(0)=(\sigma(o))^{-1} \equiv \sigma_{\min }^{-1}$.

Consider the following model. Let the functioning system be characterised by $F(x)$ and $\Phi(x)$ as in the previous section. Assume that during gradual repair the parameter monotonically increases from 0 to $\Phi(0)$ according to the stochastic process of the following specific form:

$$
\Phi_{B}(x)=\mathrm{K}(x, B),
$$

where $B$ is some positive random variable with DF $S(x)$ and for each (almost each) realisation of $B=b$ the function $\mathrm{K}(x, b)$ is monotonically increasing. Linear and exponential functions are the simplest examples:

$$
\Phi_{1 B}(x)=B x ; \quad \Phi_{2 B}(x)=\exp \{B x\}-1, \quad x \geq 0 .
$$

We are going to derive parametric availability $A_{P}(t)$ and $P\left(\Phi_{t} \geq \Phi_{0}\right)$ for the described system. Denote by $\theta_{x}(u)$ the DF of $\Phi_{B}(x)$ for the fixed $x$. Let $\xi(x)$ be the stochastic process equal to 0 for $\Phi_{B}(x) \geq \Phi(0)$ :

$$
\xi(x)=\Phi_{B}(x) \mathrm{I}\left(\Phi(0)-\Phi_{B}(x)\right) .
$$

For the fixed $x$ the corresponding mean is defined as

$$
E[\xi(x)]=\int_{0}^{\Phi(0)} u d \theta_{x}(u)
$$

and $P\left(\xi(x) \geq \Phi_{0}\right)$ as

$$
P\left(\xi(x) \geq \Phi_{0}\right)=\int_{\Phi_{0}}^{\Phi(0)} d \theta_{x}(u)=\theta_{x}(\Phi(0))-\theta_{x}\left(\Phi_{0}\right) .
$$


The DF of time of repair is defined as function of $x$ from the equation:

$$
G(x)=1-\theta_{x}\left(\Phi_{0}(0)\right) .
$$

Generalising equation (1) it is easy to obtain

$$
\begin{gathered}
A_{P}(t)=E\left[\Phi_{t}\right]=(1-F(t)) \Phi(t)+\int_{0}^{t} f(x) E[\xi(t-x)] d x \\
+\int_{0}^{t} h(x)[1-F(t-x)] \Phi(t-x) d x+\int_{0}^{t} h(x) \int_{0}^{t-x} f(y) E[\xi(t-x-y)] d y d x,
\end{gathered}
$$

where $f(x)=F^{\prime}(x)$.

Thus, equation (5) presents the general expression for parametrical availability $A_{P}(t)$. Conducting the limit transition as $t \rightarrow \infty$ :

$$
A_{S P}=\frac{1}{T+\tau} \int_{0}^{\infty}(1-F(x)) \Phi(x) d x+\frac{1}{T+\tau} \int_{0}^{\infty} E[\xi(x)] d x .
$$

Indeed, the first two terms in the right hand side of (5) vanish and the third term is defined in (2). After applying the key renewal theorem to the last term, we arrive at equation (6). It can be written in the speaking for itself form:

$$
A_{S P}=\frac{T}{T+\tau} \frac{1}{T} \int_{0}^{\infty}(1-F(x)) \Phi(x) d x+\frac{\tau}{T+\tau} \frac{1}{\tau} \int_{0}^{\infty} E[\xi(x)] d x .
$$

This result for the stationary characteristic is intuitively clear: the initial alternating renewal process can be split into two renewal processes without delay; the first one consists only of periods of operation, the second one consists only of periods of gradual repair. In doing so, $T / T+\tau$ appears to be the stationary probability to choose the first process and $\tau / T+\tau$-the second.

\section{Example 2}

Let $\Phi_{B}(x)=B x$. Then for arbitrary DF $S(x)$ ( $B$ is non-negative) the following intuitively understandable result is true:

$$
\frac{1}{\tau} \int_{0}^{\infty} E[\xi(x)] d x=\frac{\Phi_{0}}{2} .
$$

Indeed, substituting $\xi(x)=B x \mathrm{I}(\Phi(0)-B x)$ in equation (8): 


$$
E\left[\int_{0}^{\infty} B x \mathrm{I}(\Phi(0)-B x) d x\right]=E\left[\int_{0}^{\frac{\Phi(0)}{B}} B x d x\right]=E\left[\frac{\Phi(0)}{B}\right] \frac{\Phi(0)}{2} .
$$

It is clearly seen, that $E[\Phi(0) / B]$ is equal to the mean time of repair, because for the model of this example $\Phi(0) / B$ is the time of repair in every realisation $B=b$. This consideration finally leads to equation (8).

In the specific case, when $\Phi(x) \equiv \Phi(0) \equiv 1, \forall x \in[0, \infty)$, equation (6) simplifies to

$$
A_{S P}=\frac{T}{T+\tau}+\frac{1}{2} \frac{\tau}{T+\tau} .
$$

According to definition, the system's unavailability is $\tau /(T+\tau)$. This means that the possibility of gradual repair results in this example in reducing unavailability by one half and it can be quite important in practice.

Similar to equation (5) and generalising equation (3), the following formula can be obtained for the probability $P\left(\Phi_{t} \geq \Phi_{0}\right)$ :

$$
\begin{aligned}
& P\left(\Phi_{t} \geq \Phi_{0}\right)=(1-F(t)) \mathrm{I}\left(t_{0}-t\right)+\int_{0}^{t} f(x)\left[\theta_{t-x}(\Phi(0))-\theta_{t-x}\left(\Phi_{0}\right)\right] d x \\
& +\int_{0}^{t} h(x)[1-F(t-x)] \mathrm{I}\left(x-t+t_{0}\right) d x+\int_{0}^{t} h(x) \int_{0}^{t-x}\left[\theta_{t-x-y}(\Phi(0))-\theta_{t-x-y}\left(\Phi_{0}\right)\right] d x d y .
\end{aligned}
$$

Using the similar argument, as when deriving relation (6), the stationary probability is defined as

$$
P_{S P}=\frac{T}{T+\tau} \frac{1}{T} \int_{0}^{t_{0}}(1-F(x)) d x+\frac{\tau}{T+\tau} \frac{\tau-\tau_{0}}{\tau},
$$

where $\tau_{0}$ is the mean time of repair to a level $\Phi_{0}$ (while $\tau$ is the mean time of repair to the full (maximal) level $\Phi(0)$ ). When $\tau_{0}=\tau$, we arrive at the case described by the relation (4), which does not take into account the possibility of gradual repair.

Finally, it is clear that some important for practical applications generalisations are rather straightforward. For example, it is reasonable to assume that the system is available during the process of gradual repair only if $\Phi_{B}(x)$ exceeds some fixed predetermined level $\Phi_{m}$ and then to assess availability for this case. The same argument can be obviously applied to the periods of functioning as well, when the system is considered unavailable, if the performance function 
is beneath some level. In this case, however, the maintenance activities can be scheduled upon reaching this predetermined level. The question is, whether it is reasonable or not to perform maintenance in terms of cost per cycle, for instance. Using the developed approach it is easy to obtain an optimal maintenance strategy for this case.

\section{IMPERFECT REPAIR}

The results of previous sections were derived under the usual assumption that repair is ideal, thus returning the system to "as good as new" state after it. In practice repair is always imperfect and it is important to understand the errors of the model due to idealized assumptions. It is worth mentioning, that even the replacement of the failed item by the spare one cannot be considered (in the strict sense) as a perfect repair, because the spares are ageing in storage. We shall consider here only one type of imperfect repair, which is defined as the combination of the perfect repair and the minimal repair [2]. For simplicity the case of instantaneous repair will be treated when $\tau=0$. Thus, parametrical availability will be understood purely as the expected value of the corresponding function of performance.

Let with probability $p(x)$ every repair be perfect and with probability $1-p(x)$ be minimal. The standard definition of the minimal repair for the component, which had failed at time $t$ since the start of functioning, is as follows[3]:

$$
F(x \mid t)=\frac{F(x+t)-F(t)}{1-F(t)},
$$

where $F(x \mid t)$ denotes the remaining lifetime DF of the component after the minimal repair. Consider the random time between successive times of perfect repairs (moments of perfect repair form the renewal process) with DF $F_{P}(x)$. A well-known result of Beichelt and Fischer [3] is that

$$
F_{P}(x)=1-\exp \left\{-\int_{0}^{t} p(u) \lambda(u) d u\right\},
$$

where $\lambda(x)$ is the failure rate, defined by the continuous $F(x)$.

We shall generalise this approach on the case when only perfect repair (as it was in section 1) restores the function of performance to its maximal level $\Phi(0)$, while along with minimal repairs (statistically defined by (11)) this restoration is performed to the lower level to be specified later. We shall call this type of repair the minimal-imperfect repair: it is minimal, 
"concerning the DF" and imperfect, concerning the performance function. As a specific case it can be restored to the level it had just prior the failure, but we are looking at amore general situation.

Coming back to the case study of the precise navigation system, it should be pointed out that the perfect repair results in the replacement of the failed part and a highly accurate correction, using satellites, for instance. For the minimal-imperfect repair less accurate corrections from other sources are usually performed. The quality of these corrections depends on the time since the last perfect repair, thus exhibiting a certain ageing property of information.

We shall discuss for simplicity and possible practical applications only the stationary case. Equation (2) should be modified for the case under consideration:

$$
A_{S P}=\frac{1}{T_{P}} \int_{0}^{\infty} \exp \left\{-\int_{0}^{x} p(u) \lambda(u) d u\right\} E[\tilde{\Phi}(x)] d x
$$

where $\widetilde{\Phi}(x)$ is the value of the performance function in $x$ units of time after the last perfect repair (it is random now, because the minimal-imperfect repair is performed at random instants of time in the interval since the last perfect repair till current $x$ ) and $T_{P}$ is the mean of $F_{P}(x)$. The following model for $\widetilde{\Phi}(x)$ can be considered

$$
E[\widetilde{\Phi}(x)]=\exp \left\{-\int_{0}^{x} \lambda(u) d u\right\} \Phi(x)+\int_{0}^{x} \lambda(y) \exp \left\{-\int_{y}^{x} \lambda(u) d u\right\} \Phi(x, y) d y .
$$

The first term in the right hand side of equation (13) gives (with the corresponding probability) the value of the performance function when no minimal-imperfect repairs had occurred since the last perfect repair. Obviously it is the same function as in (2). The second term defines under the integral the probability that the last minimal-imperfect repair had occurred in $(y, y+d y]$ and describes the model for the value of performance function $\Phi(x, y)$ as a function of time since the last perfect repair $x$ and since the last minimal-imperfect repair $(x-y)$. Indeed, $\lambda(y) d y$ is the probability that a failure (and the minimal repair) had occurred in $(y, y+d y]$ and the exponential function under the integral defines the probability that there were no more failures in $(y, x]$. The simplest model for $\Phi(x, y)$ is

$$
\Phi(x, y)=\frac{C(y)}{\Phi(0)} \Phi(x-y),
$$


where $C(y)$ is the level of minimal-imperfect repair, performed at time $y$ after the last perfect repair. The function $C(y)$ is assumed to be monotonically decreasing and $C(y)<\Phi(y) ; y>0 ; C(0)=\Phi(0)$.

\section{Example 3}

Let $\Phi(x)=\exp \left\{-\alpha_{1} x\right\}, \quad C(y)=\exp \left\{-\alpha_{2} y\right\}, \quad \alpha_{1}>\alpha_{2}$. Then

$\Phi(x, y)=\exp \left\{-\alpha_{1} x\right\} \exp \left\{-\left(\alpha_{1}-\alpha_{2}\right) y\right\}$. Let also $\lambda(x) \equiv \lambda$ and $p(x) \equiv p$. Then, performing simple calculations, using formulas (12) and (13):

$$
A_{S P}=\frac{\lambda p}{\alpha_{1}-\alpha_{2}-\lambda}\left[\frac{\alpha_{1}-\alpha_{2}}{\lambda+\lambda p+\alpha_{1}}-\frac{\lambda}{2 \alpha_{1}-\alpha_{2}+\lambda p}\right] .
$$

If $\alpha_{1}=\alpha_{2}=\alpha$ and $p=1$, then (15) turns to $A_{S P}=\lambda /(\lambda+\alpha)$ which coincides with the result of example 1 .

Other models for $\Phi(x, y)$ can be considered as well. Similar to equation (12) the stationary probability of exceeding the fixed level $\Phi_{0}$ can be defined:

$$
P_{S P}=\frac{1}{T_{p}} \int_{0}^{\infty} \exp \left\{-\int_{0}^{x} p(u) \lambda(u) u\right\} P\left(\tilde{\Phi}(x) \geq \Phi_{0}\right) d x,
$$

where $P\left(\tilde{\Phi}(x) \geq \Phi_{0}\right)$ is obtained in the way similar to (13).

\section{CONCLUDING REMARKS}

Parametric availability is the natural generalisation of the usual binary availability. In combination with probability of exceeding the fixed level it can present all needed information on system's performance function for an arbitrary moment of time. The conventional availability shows the proportion of time when the system is operable. In parametrical case the expected level of operation is of interest and this can lead to understanding that for certain types of systems the periods of repair (when the system is down) can be not so important for the availability evaluation as they were in the binary case. That is why in section 3 we focus on considering the model for instantaneous repair.

It was assumed for simplicity that $\Phi(x)$ is a deterministic function, though the results, defining $A_{P}(t)$ and $A_{S P}$, can be easily generalised on $\Phi(x)$ being a stochastic process. The 
generalisation on obtaining $P\left(\Phi_{t} \geq \Phi_{0}\right)$ and $P_{S P}$ is not so straightforward. It can be performed without any difficulties only for the simple stochastic processes of section 2 .

The notion of gradual repair is rather fruitful for availability analyses of systems with considerable duration of repair. This type of repair, as it was stated before, usually includes adjustment (alignment) activities during which the system can be partly operable.

In this paper only one sort of imperfect repair was considered: imperfect repair, based on combination of perfect and minimal repair. Models of general repair [4,5] can be treated in the frame of this paper as well. But there exist some mathematical difficulties on this way to be investigated in the future research.

\section{REFERENCES}

[1] A. HOYLAND and M. RAUSAND, System Reliability Theory, John Wiley \& Sons, New York (1994).

[2] F. BEICHELT and K. FISCHER, General failure model applied to preventive maintenance policies. IEEE Transactions on Reliability, 29, 39-41 (1980).

[3] M.S. FINKELSTEIN, Some notes on two types of minimal repair, Advances in Applied Probability, 24, 226-229 (1992).

[4] M.S. FINKELSTEIN, The concealed age of distribution functions and the problem of general repair, Journal of Statistical Planning and Inference, 65, 315-321 (1997).

[5] M. KIJIMA, Some results for repairable systems with general repair, Journal of Applied Probability, 26, 89-102 (1989). 